\title{
Biodegradation of Certain Polycyclic Hydrocarbons with Paenbacillus Alvei and Penicillum Restricum
}

\author{
Atoke O. Ogunbayo ${ }^{1 *}$, Ogirima O. Olanipekun', Abiola H. Owoade \\ 1 Department of Chemical Engineering, University of Lagos, Yaba, Nigeria \\ * Corresponding author's e-mail: aogunbayo@unilag.edu.ng
}

\begin{abstract}
The biodegradation of polycyclic aromatic hydrocarbons (PAHs) such as naphthalene, 2-methylnaphthalene and anthracene was investigated using pure and mixed culture of Paenbacillus alvei (bacteria) and Penicillum restricum (fungi) (isolated from crude oil contaminated sites in Rivers state of Nigeria). The abilities of these organisms to biodegrade the PAHs were studied by growing the isolates in a mineral salt medium (MSM) with the PAHs in shake flasks placed in a shaking water bath rotating at $150 \mathrm{rpm}$ at room temperature for 21 days. The samples were withdrawn every three days for analysis of the residual PAHs using SRI 8610C Gas Chromatograph (GC), while the growth of the organisms was determined by using the dry biomass method. The results showed that the concentrations of PAHs decreased with an increase in the exposure time throughout a 21-day period, thus confirming the abilities of the organisms to feed on the PAHs. The results showed that the bacteria had more affinity for naphthalene, while the fungi had more affinity for anthracene. It was, however, observed that the samples from the flask which contained mixed PAHs and mixed culture of Paenbacillus alvei and Penicillum restricum had the highest and most significant biomass growth thus suggesting a synergy between the two organisms.
\end{abstract}

Keywords: biodegradation, PAHs, naphthalene, 2-methynaphthalene, anthracene, contaminated soil.

\section{INTRODUCTION}

Polycyclic aromatic hydrocarbons (PAHs) are a large group of fused-ring organic contaminants that are ubiquitous in the natural environment, and originate from two main sources, natural and anthropogenic. PAHs can be introduced to the environment through the natural sources such as volcanic eruptions and forest fires, and can also be emitted to the environment through the anthropogenic sources involving combustion of fossil fuels. These compounds are produced by industrial activities such as oil processing and are often found in contaminated soil and natural waters via wastewater effluents from coke and petroleum refining industries, accidental spills and leakages, rainwater runoff from highways and roadways, or from intentional disposal (Karthikeyan and Bhandari, 2001). They are generally referred to as "priority pollutant" because of their presence in all components of environment, resistance to- wards biodegradation, potential to bio-accumulate and carcinogenic activities (Karthikeyan and Bhandari, 2001). They have generated significant concern and have been subject of much research work over the years.

PAHs and their metabolites are reported to possess mutagenic and carcinogenic properties that affect humans and other animals (Arun and Eyini, 2011; Samanta et al., 2002), and as such, the decontamination of PAH polluted sites is of upmost importance. The investigations on the composition of PAHs found in several contaminated areas revealed that contamination is the result of a mixture of PAHs, rather than a single type of contaminant (Norramit et al., 2005; Thavamani et al., 2011). PAHs continuously increase and accumulate in the soil and water, due to the human activities that cause the emissions of these contaminants into the environment every day, and thus appropriate treatment is required to reduce the concentration and toxicity of these 
substances. It is therefore important to look into and understand the biodegradation of PAHs mixture for successful implementation of bioremediation technology. Biodegradation of a single PAH and their mixtures by degradative bacteria have been reported by several researchers (Desai et al., 2008; Wong et al., 2002; Yu et al., 2005; Yuan et al., 2000). There has been an increased interest in developing and understanding the microbial degradation processes when PAHs are present in complex mixtures, because a mixture of contaminants in a bioremediation system may result in inhibition, co-metabolism or augmentation (Hughes et al., 1997). For example, both inhibition and co-metabolism have been observed in the degradation of a mixture of phenanthrene and fluorantene by a Pseudomonas sp. (Bouchez et al., 1995). Previous studies of PAH degradation by mixed and pure cultures presented the evidence that there are interactions between PAHs in mixtures that influence biodegradation.

This paper, addresses the abilities of the indigenous microorganisms (Paenbacillus alvei and Penicillum restricum) in pure and mixed culture to degrade three PAHs (naphthalene, 2-methyl naphthalene and anthracene) individually and when mixed together. The microorganisms which were found to have biodegradation potentials for hydrocarbon from Nigerian soil were isolated from Niger Delta contaminated soil.

\section{MATERIALS AND METHOD}

All chemicals and media used in this study are of analytical grade. Naphthalene, 2-methyl naphthalene, anthracene and mineral salt media (MSM) were procured from Sigma Chemical Co. (Germany). All PAHs used have the purity $>99 \%$.

Paenibacillus alvei and Penicilium restrictum used in this study were previously isolated from Niger Delta crude oil contaminated soil. They were maintained in a slant and stored at a temperature of $4^{\circ} \mathrm{C}$. The stock was used to prepare seed cultures for all the experiments. For inoculation, the stock was cultivated on nutrient broth for three days, and the cells were harvested by centrifugation and washed twice with phosphate buffer (50 $\mathrm{mM}, \mathrm{pH}$ 7.2). The cells were re-suspended in the same buffer and used as the starter culture for the experiments. The mineral salts medium (MSM) used for the biodegradation experiment contained the following composition in gram per litre $(\mathrm{g} / \mathrm{l})$ :
$\mathrm{NH}_{4} \mathrm{NO}_{3}, 12 \mathrm{~g} ; \mathrm{Na}_{2} \mathrm{HPO}_{4}, 6.0 \mathrm{~g} ; \mathrm{KH}_{2} \mathrm{PO}_{4}, 3.18 \mathrm{~g}$ $\mathrm{MgSO}_{4}, 7 \mathrm{H}_{2} \mathrm{O}, 0.60 \mathrm{~g} ; \mathrm{K}_{2} \mathrm{SO}_{4}, 1.02 \mathrm{~g}$; which was prepared by dissolving the salts in distilled water. The medium was sterilized by autoclaving at $121^{\circ} \mathrm{C}$ for 20 minutes.

A loop was transferred from the stock culture to a $100 \mathrm{ml}$ conical flask containing $100 \mathrm{ml}$ MSM and $1 \%(\mathrm{v} / \mathrm{v})$ of each PAH as sole carbon source. This was done separately for each PAH with pure culture of the organisms. The six setups (primary cultures) were incubated in a shaker at $120 \mathrm{rpm}$ for 24 hours.

After 24 hours, the secondary cultures were prepared by transferring $50 \mathrm{ml}$ of the cultures to $50 \mathrm{ml}$ measured MSM and incubated in $100 \mathrm{ml}$ conical flask in the rotary shaker at $120 \mathrm{rpm}$ for 12 hours at $30^{\circ} \mathrm{C}$, and the inoculums for the experiments were taken from secondary cultures.

The biodegradation abilities of each of the microorganisms (Paenibacillus alvei and Penicilium restrictum) and the mixture of the two were examined on each of the PAHs being studied (naphthalene, 2-methyl naphthalene and anthracene) alone and in a mixture. The experiment was set up in 13 flasks, the spouts of which were covered with cotton wool. Each flask containing $4 \mathrm{ml}$ of the relevant PAHs, and $186 \mathrm{ml}$ of sterile mineral salt medium, was inoculated with 10 $\mathrm{ml}$ of the inoculum and placed in the incubator shaker rotating at $150 \mathrm{rpm}$ at room temperature for 21 days. A control sample flask containing the PAHs and MSM but no inoculum was also set up. The samples were withdrawn initially at 3 days interval and later at 6 days for analysis.

\section{Analytical methods}

Dry biomass weight analysis was used to monitor biomass growth. A $10 \mathrm{ml}$ sample was withdrawn from each flask and centrifuged at $200 \mathrm{rpm}$ for 20 minutes. The supernatant was decanted into McCartney bottle and the residue was washed with an equal volume of distilled water, poured into pre-weighed filter paper and allowed to drain. The filter paper was dried to a constant weight in the oven at a temperature of $60^{\circ} \mathrm{C}$. The difference in the initial and dried weights of the filter paper was recorded as the dry biomass weight. This process was repeated for all samples taken for the duration of the experiments.

The efficiency of the isolated microorganisms to degrade naphthalene, 2-methyl naphthalene and anthracene in pure and mixed cultures of in- 
dividual and mixed PAHs and the corresponding growth of the organism was monitored by estimating the residual chemicals. Residual hydrocarbons in the supernatant obtained from the centrifugation were extracted by mixing with equal volume of n-hexane, and the Gas Chromatograph (GC) SRI 8610C was used to identify and quantify the PAHs. The GC was operated using the Restek 15 m length MXT-1 column and Helium as carrier gas, coupled with a printer (HP DeskJet 1515) and interfaced with a laptop (HP Compact 615). During analysis, 1 micro litre of the prepared extracts of each solute sample was injected into the GC to identify and quantify the PAHs.

\section{RESULTS AND DISCUSSION}

\section{Biodegradation of PAHs}

Pure and mixed cultures of Paenibacillus alvei and Penicilium restrictum were assessed for their potential to grow on naphthalene, 2-methyl naphthalene and anthracene alone and when mixed.

Figures 1 to 5 graphically represent the result of the biodegradation of the PAHs by pure and mixed culture of Paenibacillus alvei and Penicilium restrictum, after 21 days. The results showed that the organisms in pure state were capable of biodegrading each of the PAHs to various degrees. While the bacteria had more affinity for naphthalene and anthracene (Figure 1), the fungus had more affinity for anthracene (Figure 2).

The three PAHs mixed together were subjected to biodegradation by pure and mixed cultures, and the results are as shown in Figures 3, 4 and 5. The biodegradation of the mixed PAHs by the mixed culture showed that naphthalene and anthracene were degraded (Figure 3).

In the biodegradation of the mixed PAHs by pure culture of Penicilium restrictum as shown in Figure 4, it was observed that anthracene was the least degraded, while naphthalene was degraded to the greatest degree. In the comparison

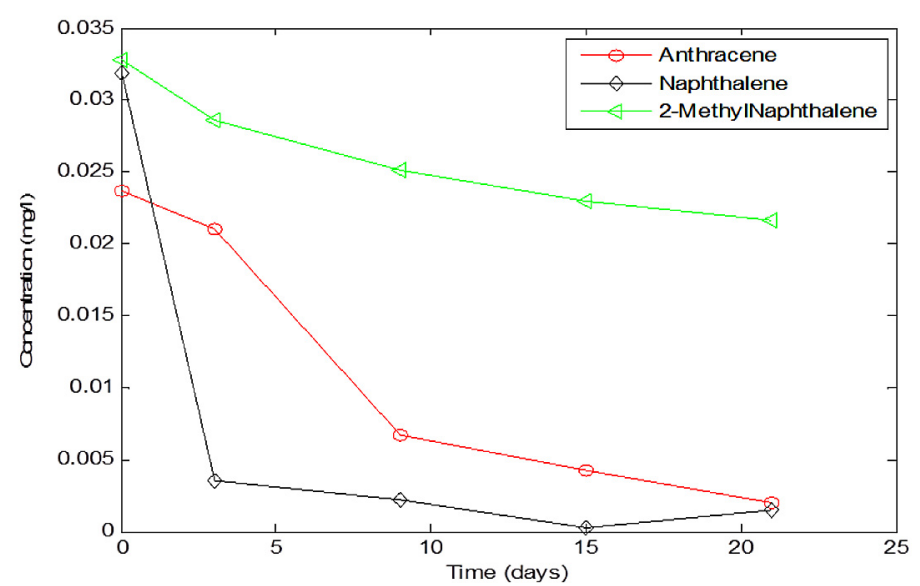

Figure 1. Plot of residual Substrate concentration of growth of Paenibacillus alvei on Naphthalene, 2-methyl naphthalene and anthracene

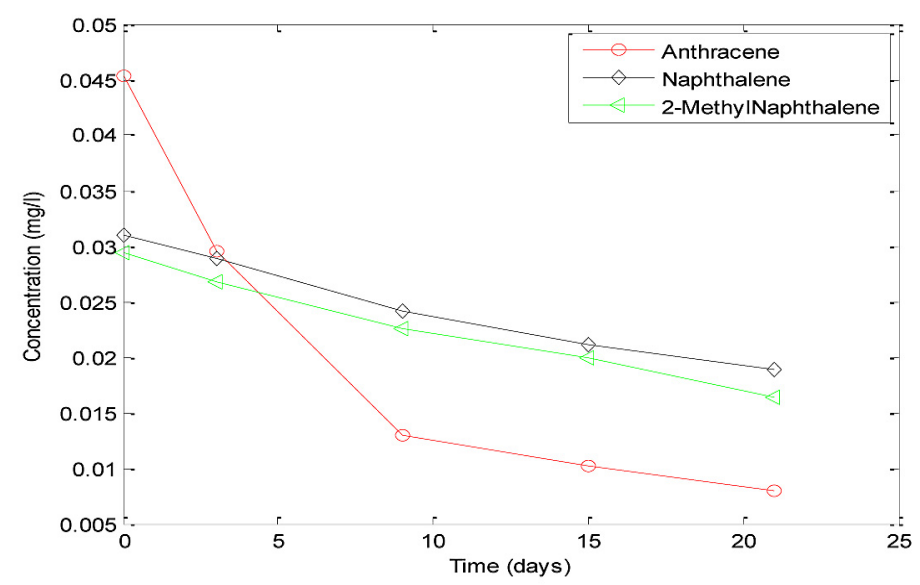

Figure 2. Plot of residual Substrate concentration of growth of Penicilium restrictum on Naphthalene, 2-methyl naphthalene and anthracene 


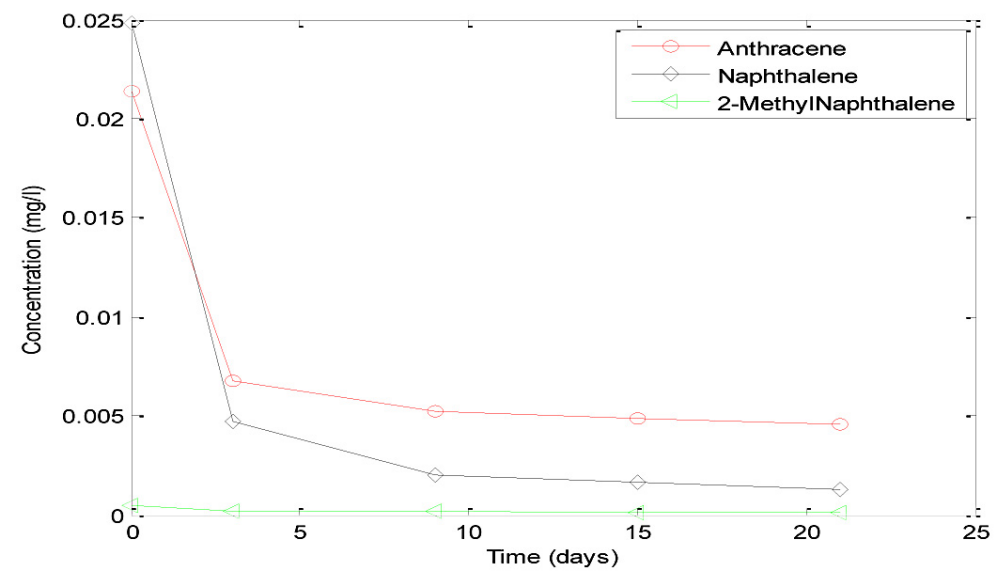

Figure 3. Plot of residual Substrate concentration of growth of mixed culture of Paenibacillus alvei and Penicilium restrictum on a mixed substrate of Naphthalene, 2-methyl naphthalene and anthracene

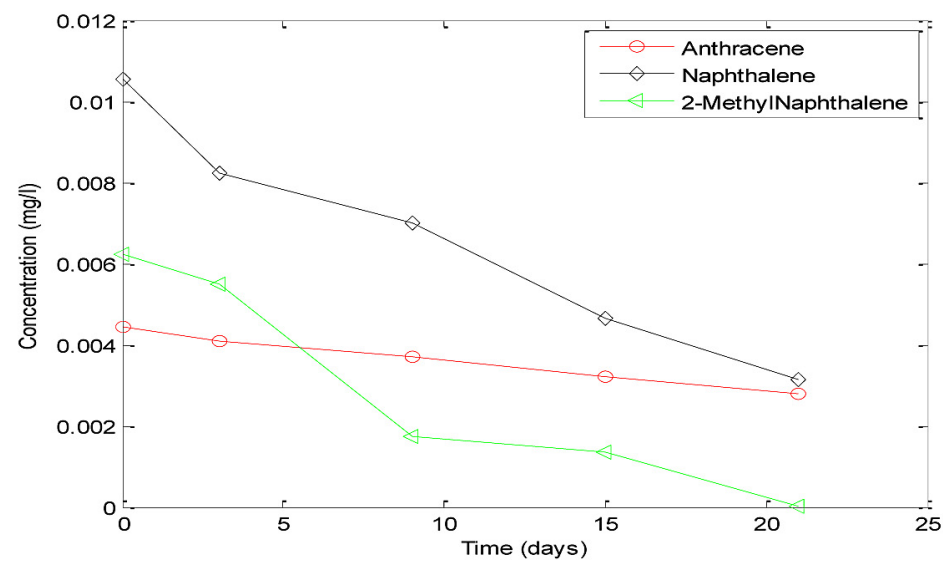

Figure 4. Plot of residual Substrate concentration of growth of pure culture of Penicilium restrictum on a mixed substrate of Naphthalene, 2-methyl naphthalene and anthracene

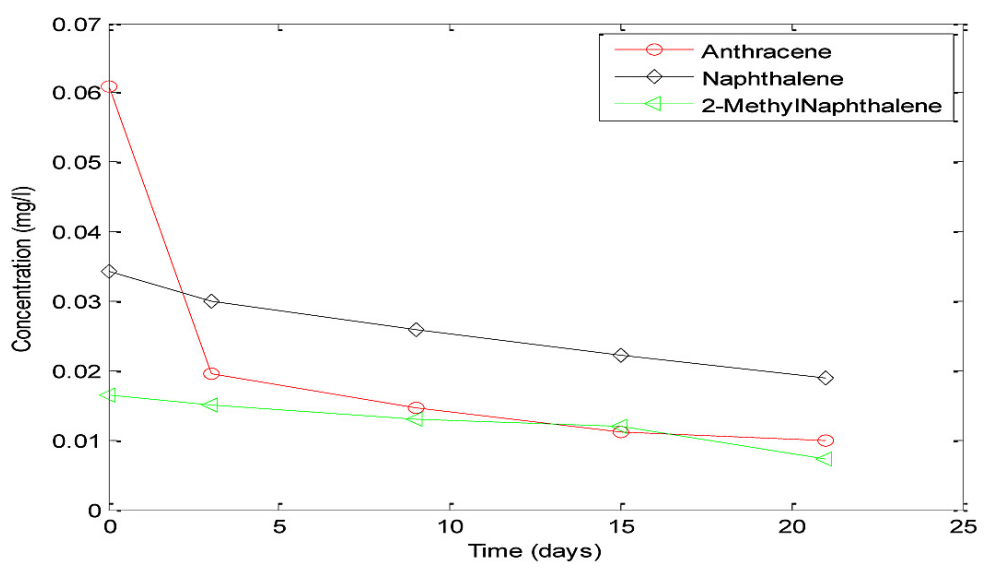

Figure 5. Plot of residual Substrate concentration of growth of pure culture of Paenibacillus alvei on a mixed substrate of Naphthalene, 2-methyl naphthalene and anthracene

involving the results of the individual PAHs by pure culture of Penicilium restrictum where anthracene was better degraded, this result showed that Penicilium restrictum had more affinity for naphthalene when the substrates are mixed. Similarly, in the biodegradation of the mixed PAHs by pure culture of Paenibacillus alvei as shown in Figure 5, it was observed that anthracene was better degraded.

Figures 6 and 7 showed the result of the control experiment for the biodegradation of PAHs after 21 days for anthracene, naphthalene and 


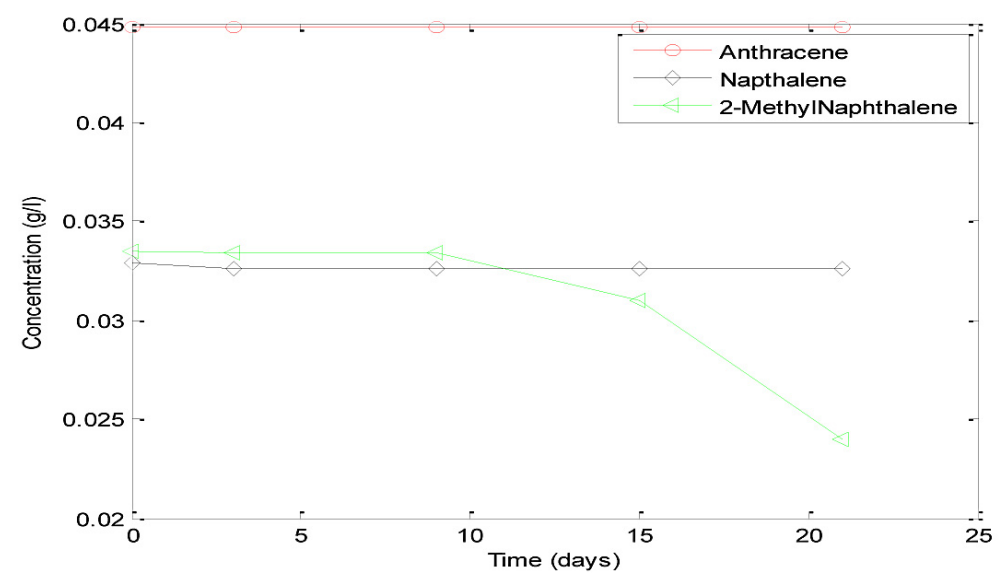

Figure 6. Plot of residual Substrate concentration of Naphthalene, 2-methyl naphthalene and anthracene in control set-up

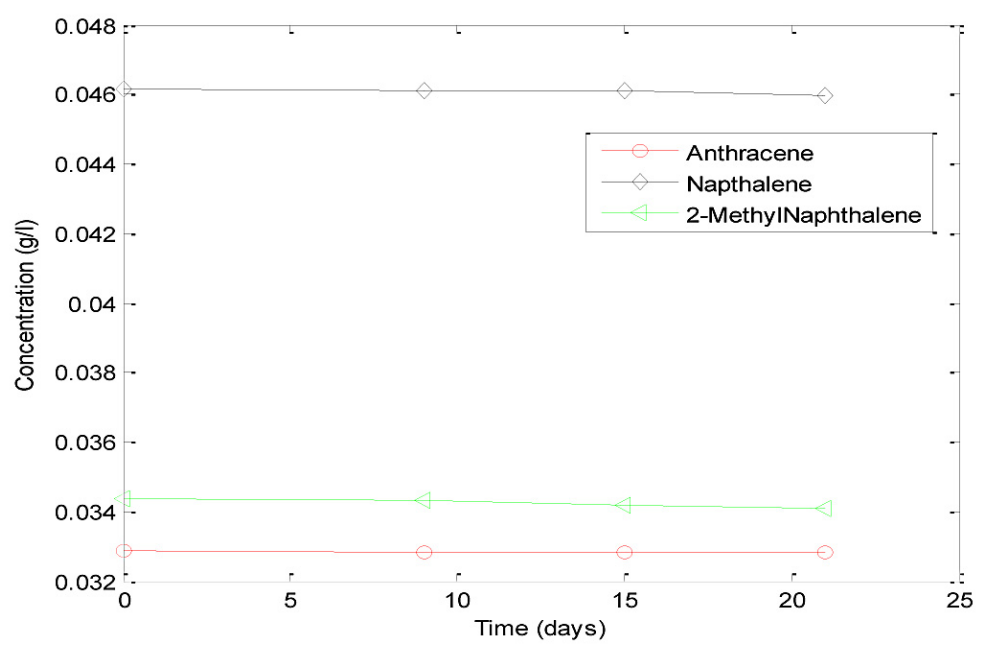

Figure 7. Plot of residual Substrate concentration of mixed substrate of Naphthalene, 2-methyl naphthalene and anthracene in control set-up

2-methyl naphthalene (with no microorganisms). The biodegradation tests results showed slight decrease in the initial concentration of the polycyclic aromatic hydrocarbons (PAHs) used. This could be as a result of abiotic factors.

\section{Substrate degradation and growth rate}

Figures 8 to 16 showed graphically the results of the degradation of the individual and mixed PAHs and growth rate of the microbes. From the experimental result, it was observed that the two microbes, Paenibacillus alvei and Penicilium restrictum grew and degraded the PAHs under the experimental conditions. It was observed that as the biomass increased, the concentration of the PAHs decreased. This implies that the concentration of PAHs used in this study was not toxic to the degrading microorganisms, and the organisms were able to degrade them and feed on them. The comparison pertaining to the abilities of the microbes to degrade the PAHs,

Table 1. Kinetic parameters

\begin{tabular}{|l|c|c|}
\hline \multicolumn{1}{|c|}{$\begin{array}{c}\text { Microorganism / } \\
\text { Substrate }\end{array}$} & $\mu_{\max }(\mathrm{g} / \mathrm{l})$ & $\mathrm{K}_{\mathrm{M}}$ \\
\hline $\begin{array}{l}\text { BA - Bacteria/ } \\
\text { Anthracene }\end{array}$ & 0.0160 & 0.0327 \\
\hline $\begin{array}{l}\text { BN - Bacteria/ } \\
\text { Naphthalene }\end{array}$ & 0.0670 & 0.000375 \\
\hline $\begin{array}{l}\mathrm{BM} \text { - Bacteria/2- } \\
\text { methylnaphthalene }\end{array}$ & 0.0160 & 0.0327 \\
\hline FA - Fungi/Anthracene & 0.0294 & 0.00881 \\
\hline $\begin{array}{l}\text { FN - Fungi/ } \\
\text { Naphthalene }\end{array}$ & 0.02935 & 0.03564 \\
\hline $\begin{array}{l}\text { FM - Fungi/ } \\
\text { 2-methyNaphthalene }\end{array}$ & 0.03277 & 0.03478 \\
\hline $\begin{array}{l}\mathrm{YX}-\text { Mixed PAHs On } \\
\text { Mixed Organism }\end{array}$ & 0.02872 & 0.01591 \\
\hline $\begin{array}{l}\text { FX - Mixed PAHs on } \\
\text { fungi }\end{array}$ & 0.01818 & 0.00769 \\
\hline $\begin{array}{l}\mathrm{BX}-\text { Mixed PAHs on } \\
\text { Bacteria }\end{array}$ & 0.03030 & 0.000243 \\
\hline
\end{tabular}




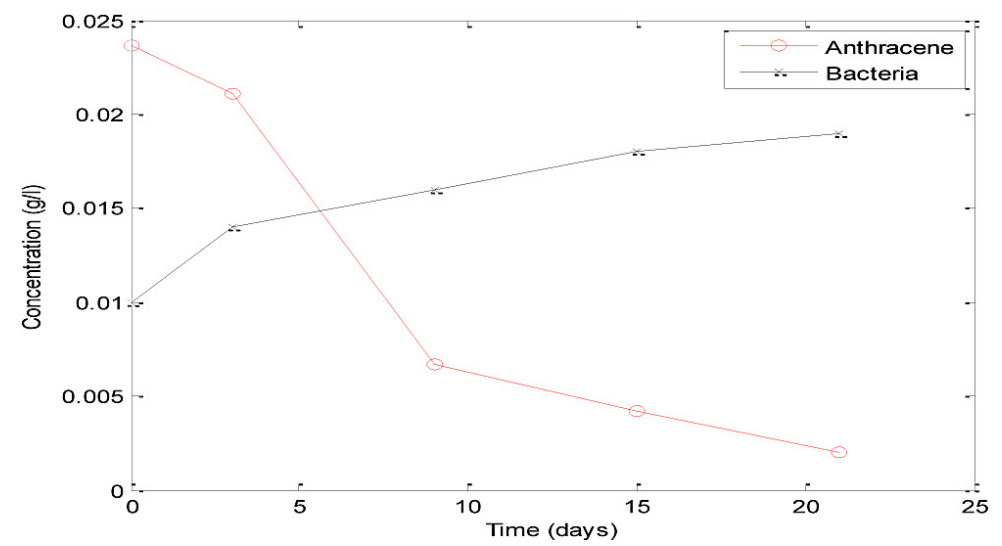

Figure 8. Plot of Biomass concentration and substrate utilization of growth of Paenibacillus alvei on Anthracene.

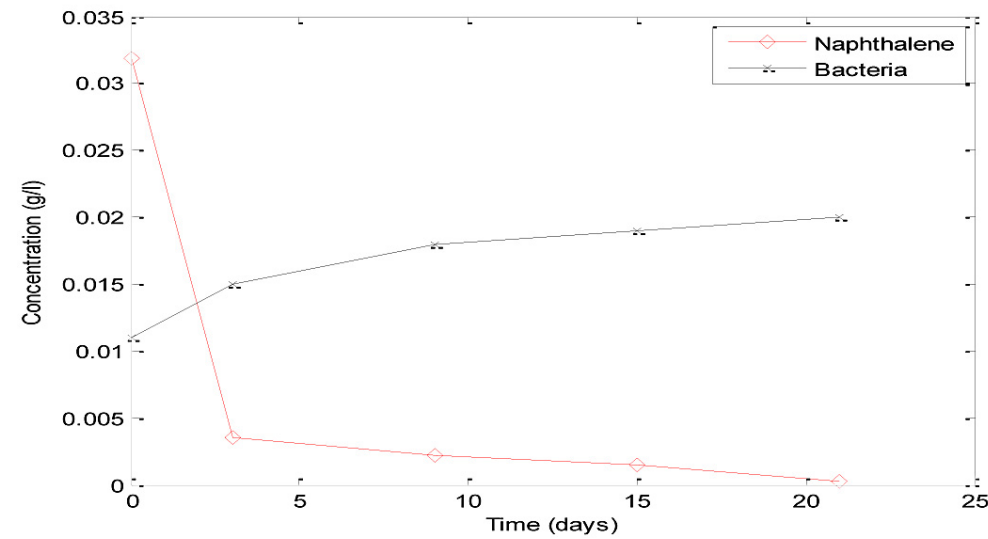

Figure 9. Plot of Biomass concentration and substrate utilization of growth of Paenibacillus alvei on Naphthalene

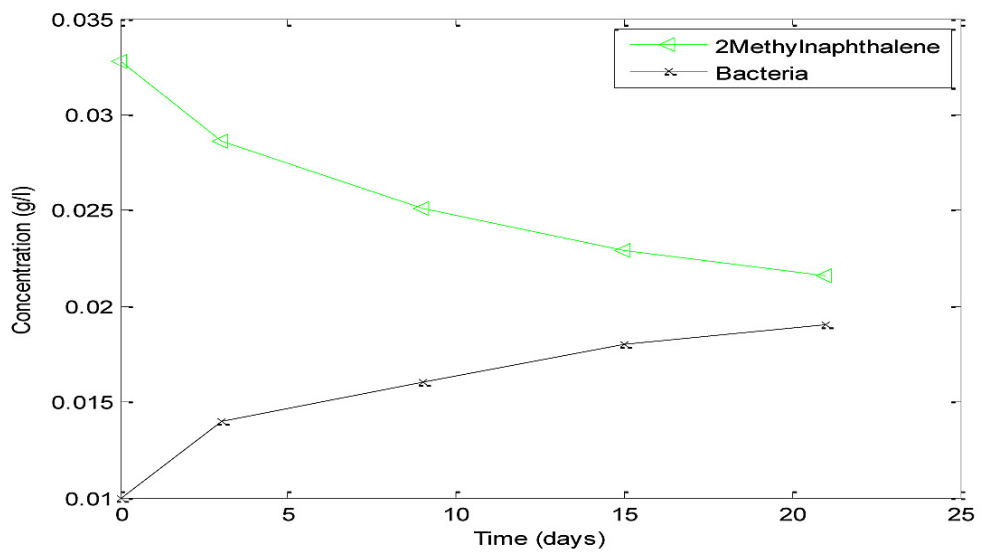

Figure 10. Plot of Biomass concentration and substrate utilization of growth of Paenibacillus alvei on 2-methylnaphthalene.

showed that while anthracene and 2-methylnaphthalene were better degraded by Penicilium restrictum than by Paenibacillus alvei, naphthalene was better degraded by Paenibacillus alvei than by Penicilium restrictum. The highest growth of $0.03 \mathrm{~g} / 1$ was observed with mixed PAHs and mixed culture (Figure 14).

\section{Kinetic parameters}

From Monod model (equation 1), $\mu_{\max }$ (maximum specific growth rate) and $\mathrm{K}_{\mathrm{m}}$ (Monod constant) can be obtained as shown in appendix B. Table 1 shows that the maximum growth rate of fungi on anthracene was more than the maxi- 


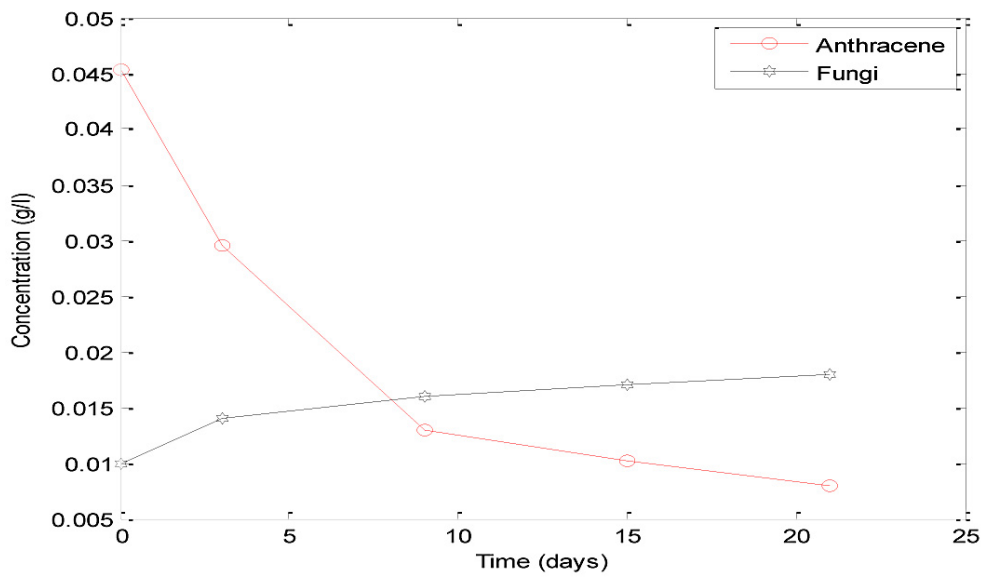

Figure 11. Plot of Biomass concentration and substrate utilization of growth of Penicilium restrictum on Anthracene

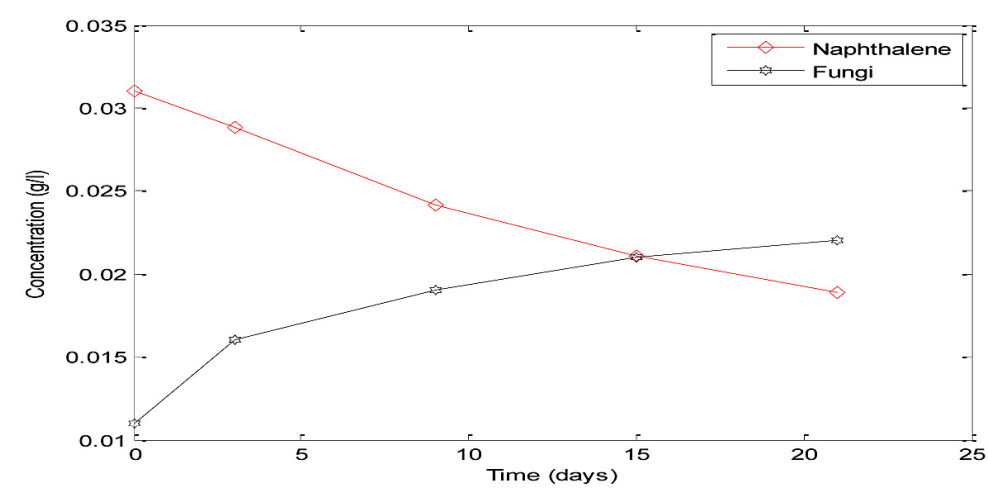

Figure 12. Plot of Biomass concentration and substrate utilization of growth of Penicilium restrictum on Naphthalene

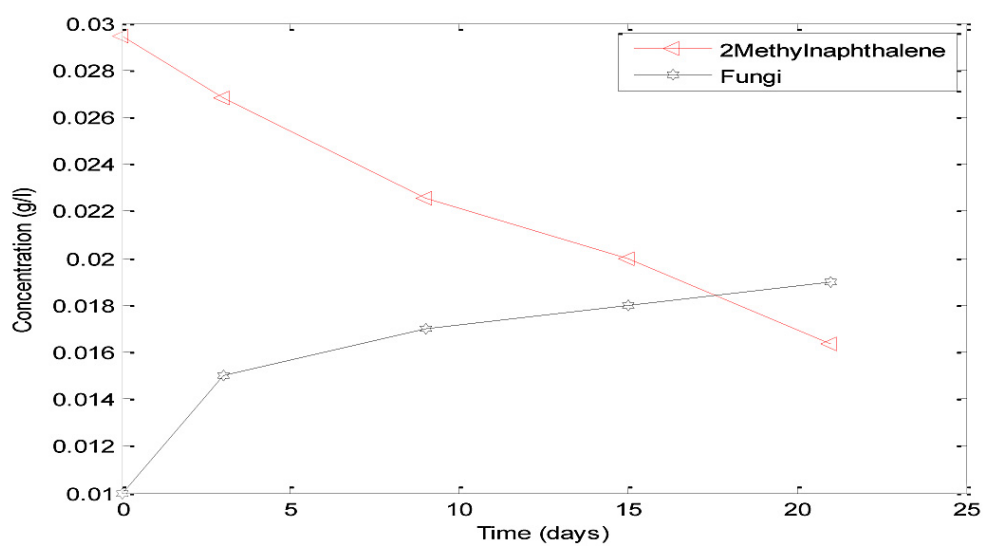

Figure 13. Plot of Biomass concentration and substrate utilization of growth of Penicilium restrictum on 2-methylnaphthalene

mum growth rate of bacteria on anthracene, while for naphthalene, the maximum growth rate of bacteria was more than the maximum growth rate of fungi and for 2-methyl naphthalene, the maximum growth rate of fungi was more than the maximum growth rate of bacteria. Pure culture of Paenibacillus alvei on naphthalene had highest growth rate.

\section{CONCLUSION}

Biodegradation of the PAHs (naphthalene, 2-methylnaphthalene and anthracene) by pure and mixed culture of bacteria and fungi was successfully carried out. Paenibacillus alvei and Penicilium restrictum both in pure and mixed cultures exhibited the abilities to biodegrade the PAHs. 


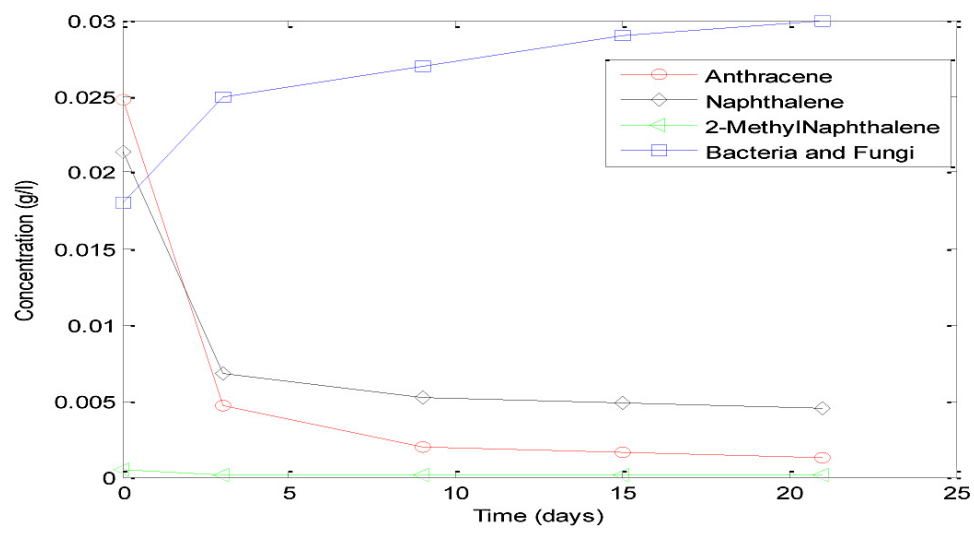

Figure 14. Plot of Biomass concentration and substrate utilization of growth of mixed culture of Paenibacillus alvei and Penicilium restrictum on a mixed substrate of Naphthalene, 2-methyl naphthalene and anthracene

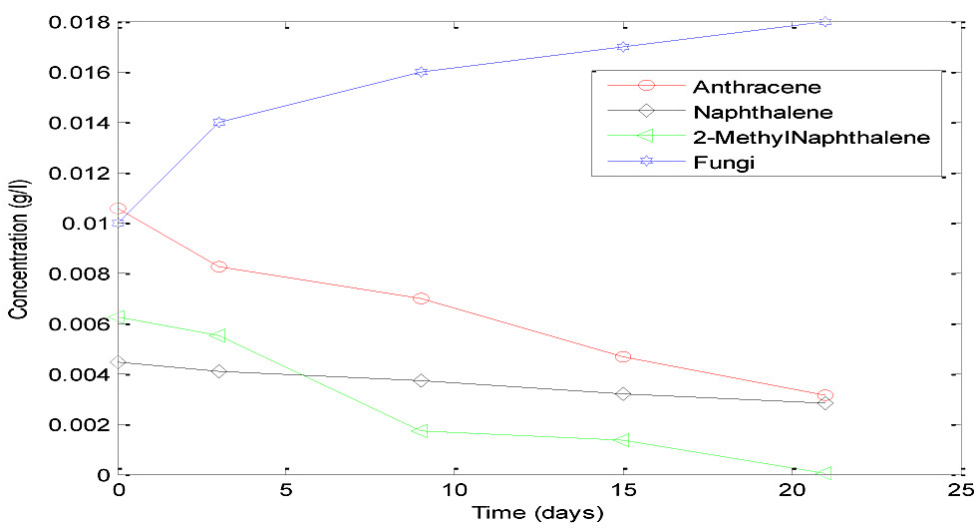

Figure 15. Plot of Biomass concentration and substrate utilization of growth of pure culture of Penicilium restrictum on a mixed substrate of Naphthalene, 2-methyl naphthalene and anthracene.

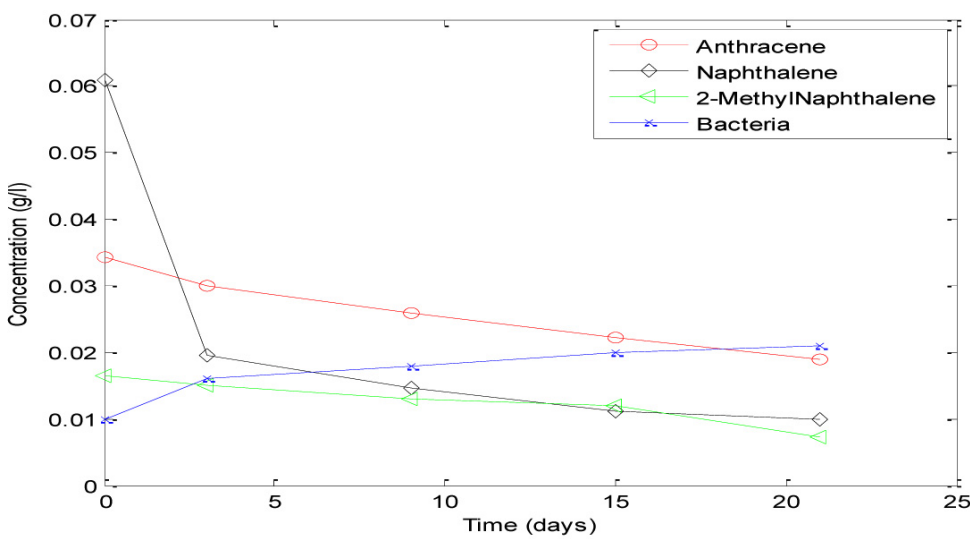

Figure 16. Plot of Biomass concentration and substrate utilization of growth of pure culture of Paenibacillus alvei on a mixed substrate of Naphthalene, 2-methyl naphthalene and anthracene.

Pure culture of Paenibacillus alvei degraded all the PAHs but it had more affinity for naphthalene than anthracene and 2-methylnaphthalene, while Penicilium restrictum had more affinity for anthracene than naphthalene and 2-methylnaphthalene. The mixed culture of Paenibacillus alvei and Penicilium restrictum degraded naphthalene better than the other components (anthracene and 2-methylnaphthalene). The kinetic parameter calculations showed that pure culture of Paenibacillus alvei on naphthalene had highest growth rate.

The mixed cultures of Paenibacillus alvei and Penicilium restrictum performed better than either of the pure cultures. It can be con- 
cluded that the mixed culture of bacteria and fungi used in this study are capable of degrading the PAHs, and it also demonstrates that the mixed culture of indigenous bacteria and fungi can be effectively used to degrade PAHs, especially naphthalene.

\section{REFERENCES}

1. Arun and Eyini, 2011. Comparative studies on lignin and polycyclic aromatic hydrocarbons degradation by basidiomycetes fungi. Bioresource Tech 102, 8063-70.

2. ATSDR. 1995. Toxicological profile for polycyclic aromatic hydrocarbons (PAHs). Agency for Toxic Substances and Disease Registry U.S. Department of Health and Human Services, Public Health Service. Atlanta, GA. Available on-line at: http:// www.atsdr.cdc.gov/tfacts69.html

3. Bouchez M., Blanchet D., Vandecas-Teele J.P. 1995. Degradation of polycyclic aromatic hydrocarbons by pure strain and by defined strain association: Inhibition phenomena and cometabolism. Appl. Microbiol. Biotechnol., 43, 156-164.

4. Desai, 2005. Biodegradability of selected polycyclic aromatic hydrocarbons (PAH) mixture. Master thesis Texas A\&M University

5. Farinazleen Mohamad Ghazali, Raja Noor Zaliha Abdul Rahman Abu Bakar Salleh, Mahiran Basri 2004. Biodegradation of hydrocarbons in soil by microbial consortium. International Biodeterioration \& Biodegradation, 54, 61-67.

6. Hughes J.B., Beckles D.M., Chandra S.D., Ward C.H. 1997. Utilization of bioremediation for the treatment of PAHcontaminated sediments. J. Ind. Microbiol. Biotech., 18, 152-160.

7. Igwo-Ezikpe M.N., Gbenle O.G., Ilori M.O., Ok- puzor J., Osuntoki A.A. 2010. High Molecular Weight Polycyclic Aromatic Hydrocarbons Biodegradation by Bacteria Isolated from Isolated from Contaminated Soils in Nigeria. Research Journal of Environmental Sciences, 4(2), 127-137.

8. Janbandhu A. and Fulekar, 2011. Biodegradation of Phenanthrene using adapted microbial consortium isolated from petrochemical contaminated environment. J. Hazard. Mater., 187, 333-340.

9. Karthikeyan and Bhandari, 2001. Anaerobic biotransformation of aromatic and polycyclic aromatic hydrocarbons in soil microcosms: A review. J. Hazard. Subst. Res. 3, 1-7.

10. Norramit, Cheevaporn, Itoh and Tanaka, 2005. Characterization and carcinogenic risk assessment of polycyclic aromatic hydrocarbons in the respirable fraction fairborne particles in the Bangkok metropolitan area. J Health Sci, 51, 437-446.

11. Samanta S.K, Singh O.V. and Jain R.K., 2002. Polycyclic aromatic hydrocarbons: Environmental pollution and bioremediation. Trends Biotechnol., 20, 243-248.

12. Thavamani, Megharaj, Krishnamurti, McFarland, and Naidu, 2011. Finger printing of mixed contaminants from former manufactured gas plant (MGP) site soils: Implications to bioremediation. Environ. Int. 37, 184-189.

13. Yu, Ke, Wong \& Tam, 2005. Degradation of polycyclic aromatic hydrocarbons (PAHS) by a bacterial consortium enriched from mangrove sediments. Environment International, 31(2), 149-154.

14. Yuan, Wei \& Chang, 2000. Biodegradation of polycyclic aromatic hydrocarbons by a mixed culture. Chemosphere, 41, 1463-1468.

15. Wong, Lai, Wan, Ma \& Fang, 2002. Isolation and optimization of PAH degradative bacteria from contaminated soil for PAHs bioremediation. Water, Air and Soil Pollution, 139, 1-13. 